\title{
Comparison of Analgesic Efficacy of Erector Spinae Plane Block and Posterior Quadratus Lumborum Block in Laparoscopic Liver Resection: A Randomized Controlled Trial
}

\author{
RyungA Kang ${ }^{1, *}$ \\ Seungwon Lee ${ }^{l, *}$ \\ Gaab Soo Kim ' \\ Ji Seon Jeong $\mathbb{D}^{\prime}$ \\ Mi Sook Gwak' \\ Jong Man Kim ${ }^{2}$ \\ Gyu-Seong Choi ${ }^{2}$ \\ Yoon Jee Cho' \\ Justin Sangwook Ko' \\ 'Department of Anesthesiology and Pain \\ Medicine, Samsung Medical Center, \\ Sungkyunkwan University School of \\ Medicine, Seoul, Korea; ${ }^{2}$ Department of \\ Surgery, Samsung Medical Center, \\ Sungkyunkwan University School of \\ Medicine, Seoul, Korea \\ *These authors contributed equally to \\ this work
}

\begin{abstract}
Purpose: Ultrasound-guided fascial plane blocks, including the erector spinae plane (ESP) and quadratus lumborum (QL) blocks, provide effective postoperative abdominal analgesia. However, there is limited evidence on the analgesic efficacy of ESP and QL blocks after liver surgery. Therefore, we aimed to compare the cumulative opioid consumption between the ESP and QL blocks in patients with hepatocellular carcinoma undergoing laparoscopic liver resection.
\end{abstract}

Patients and Methods: Eighty-eight patients scheduled to undergo laparoscopic liver resection were randomized to receive bilateral single injection of ESP block at T8 (ESP group) or bilateral single injection of posterior QL block (QL group; $20 \mathrm{~mL}$ of $0.375 \%$ ropivacaine for each side, ie, total $150 \mathrm{mg}$ of ropivacaine), in addition to intravenous (IV) fentanyl patient-controlled analgesia and multimodal analgesia. The primary outcome was cumulative opioid consumption over the first $24 \mathrm{~h}$, expressed as IV morphine equivalents. Secondary outcomes included serial plasma ropivacaine concentrations, pain scores, time to first flatus, and Quality of Recovery-15 scores.

Results: Eighty-five patients were analyzed (ESP group, $\mathrm{n}=42$; QL group, $\mathrm{n}=43$ ). Cumulative 24-h opioid consumption was similar between the ESP and QL groups (41.4 \pm $22.6 \mathrm{mg}$ vs $44.2 \pm 20.0 \mathrm{mg}$, mean difference (QL-ESP), $2.8 \mathrm{mg}, 95 \%$ confidence interval, -6.4 to $12 \mathrm{mg}, \mathrm{p}>0.99$ ). There were no significant differences in resting pain scores at 24 , 48 and $72 \mathrm{~h}$ postoperatively or recovery outcomes. The peak plasma ropivacaine concentration 30 min after injection was significantly higher in the ESP group $(1.5 \pm 0.3 \mu \mathrm{g} / \mathrm{mL})$ than in the QL group $(1.3 \pm 0.5 \mu \mathrm{g} / \mathrm{mL}, \mathrm{p}=0.035)$; however, both were lower than the arterial threshold value of systemic toxicity $(4.3 \mu \mathrm{g} / \mathrm{mL})$.

Conclusion: ESP and QL blocks provided similar postoperative analgesia in patients undergoing laparoscopic liver resection.

Keywords: regional anesthesia, erector spinae plane block, laparoscopic liver resection, quadratus lumborum block, ropivacaine

\section{Introduction}

Laparoscopic liver resection is increasingly used in patients with hepatocellular carcinoma (HCC). ${ }^{1}$ Although laparoscopic surgery is minimally invasive, a significant number of patients continue to experience moderate to severe pain after laparoscopic surgery. ${ }^{2}$ Postoperative pain after laparoscopic liver resection is complex and multifactorial, consisting of both somatic and visceral pain caused by
Correspondence: Justin Sangwook Ko Department of Anesthesiology and Pain Medicine, Samsung Medical Center, Sungkyunkwan University School of Medicine, 8I Irwon ro, Gangnam gu, Seoul, 0635I, Korea

Tel +82-2-34I0-2454

Fax +82-2-34I 0-036I

Email jsko@skku.edu 
gas distension of the abdominal wall, port placement, and dissection in the abdominal cavity. ${ }^{3,4}$ Therefore, analgesic modalities should provide upper and lower abdominal analgesia, including epigastric area, umbilical area as well as the lower abdomen. Previous studies have evaluated analgesic strategies including intrathecal morphine administration and single-injection erector spinae plane (ESP) block in laparoscopic liver resection, ${ }^{3,4}$ but there is limited evidence to draw relevant conclusions mandating effective analgesic management.

Numerous studies have reported that the erector spinae plane (ESP) block can be a potentially valuable regional anesthesia technique in various abdominal surgeries performed at a lower thoracic vertebral level (T7 or T8). ${ }^{4-8}$ Furthermore, the relatively safe profile of ESP block, including the absence of major blood vessels and neural structures, can be advantageous to other invasive techniques such as thoracic epidural analgesia or paravertebral blocks, especially when postoperative coagulopathy is a major concern after liver surgery. However, the analgesic duration and efficacy remain inconsistent after singleinjection ESP blocks in liver surgery. ${ }^{3,4}$ In addition, the risk of local anesthetic systemic toxicity can pose even greater concern in these subset of patients after the removal of substantial volume of liver where extensive local anesthetic metabolism takes place. Therefore, we sought to identify alternative analgesic modalities with favorable analgesic efficacy and safety profiles.

The quadratus lumborum (QL) block is an abdominal trunk block. Out of the three possible approaches of QL blocks (lateral, posterior, or anterior), posterior or anterior QL block provides widespread (T4 to T12 or L1) and long-lasting (up to $48 \mathrm{~h}$ ) upper and lower abdominal analgesia. ${ }^{9,10}$ Recent studies have shown that anterior QL block can reduce opioid consumption and pain scores in patients undergoing liver resection. ${ }^{11,12}$ Therefore, we expected that posterior or anterior QL block would be suitable analgesic option for liver surgery. However, the analgesic efficacy of posterior QL block in liver surgery has not been compared with that of ESP block. Therefore, we conducted a randomized trial to test the hypothesis that, bilateral posterior QL block would improve the quality of postoperative analgesia in the first $24 \mathrm{~h}$ after laparoscopic liver resection compared to that of ESP block. Furthermore, we measured the chronological changes in arterial ropivacaine concentrations in both block groups to determine the safety of the local anesthetic dose in patients.

\section{Patients and Methods}

\section{Study Participants}

This study was conducted in accordance with the Declaration of Helsinki. After receiving approval from the Samsung Medical Center Research Ethics Board (SMC 2020-01-035-006), this trial was prospectively registered with the Clinical Research Information Service (CRiS) trial registry of South Korea (identifier: KCT0005077; date of registration: 02/06/2020; principal investigator's name: RyungA Kang). Written informed consent was obtained from all participants. We enrolled adult patients with American Society of Anesthesiologists physical status classifications I-III scheduled for elective laparoscopic liver resection, including segmentectomy, right anterior sectionectomy, left lateral sectionectomy, right or left hepatectomy, or right or left extended hemihepatectomy between December 2020 and July 2021 at Samsung Medical Center, Seoul, Korea. We excluded patients who refused to participate in the study, had contraindications to peripheral nerve block, had systemic infectious disease, or had an allergy to local anesthetics in the standardized postoperative analgesic regimen.

\section{Randomization and Blinding}

A hospital staff member who was not otherwise involved in the study performed computer-generated block randomization (www.randomizer.org) in a 1:1 ratio to allocate enrolled patients to an ESP group $(\mathrm{n}=44)$ or a QL group $(n=44)$. The randomization number and allocation of each patient were concealed in a password-protected log file. All blocks were performed by a single investigator who was experienced in the technique (RyungA Kang). ${ }^{4}$ This investigator was not involved in the outcome assessment or further patient management. Study participants and the outcome assessor (Seungwon Lee) were blinded to group allocation. To maintain the blinding of the participants, all blocks were performed after induction of general anesthesia. All methods were carried out in accordance with relevant guidelines and regulations.

\section{Erector Spinae Plane Block or Quadratus Lumborum Block}

In the ESP group, the patients were placed in the right lateral position after induction of general anesthesia. Ultrasound-guided bilateral ESP blocks at the level of the T8 transverse process were performed as previously described. ${ }^{4,8}$ Using a high-frequency (6 to $13 \mathrm{MHz}$ ) linear 
probe, T8 transverse process was identified and the left ESP block (upper side) was performed first. A 21-gauge, 100-mm echogenic needle (SonoPlex STIM, PAJUNK, Geisingen, Germany) was inserted in-plane in a cranialto-caudal direction to contact the $\mathrm{T} 8$ transverse process. The correct needle tip position was confirmed by a linear pattern of injectate spread lifting the erector spinae muscle off the transverse process and $20 \mathrm{~mL}$ of $0.375 \%$ ropivacaine was injected through the needle. The right ESP block was performed in an identical manner without patient repositioning.

In the QL group, ultrasound-guided bilateral posterior QL block was performed with the patients in the supine position after the induction of general anesthesia. The reason for choosing the posterior QL block out of the three possible approaches (lateral, posterior, or anterior) was because it was indicated for any abdominal surgery above and below umbilicus with wide dermatomes coverage from $\mathrm{T} 4$ to $\mathrm{T} 12$ or $\mathrm{L} 1 .^{10}$ In addition, posterior QL block is associated with reduced pain scores after laparoscopic ovarian surgery ${ }^{9}$ and reduced opioid requirements after cesarean section when compared to placebo ${ }^{13}$ or transverse abdominis plane block. ${ }^{14}$ If necessary, a thin pillow was placed under the patient's hip, or an operating table was laterally tilted to obtain a better ultrasound image. Using a highfrequency linear probe or a convex probe (X-porte; SonoSite, Bothell, Washington, USA), the three muscle layers of the lateral abdomen; external oblique, internal oblique, and transversus abdominis muscles, and the quadratus lumborum muscle were visualized. The same echogenic needle that was used for the ESP block was inserted in-plane using an anterior-to-posterior trajectory. A local anesthetic ( $20 \mathrm{~mL}$ of $0.375 \%$ ropivacaine) was injected on the posterior surface of $\mathrm{QL}$ muscle (ie, posterior QL block). ${ }^{10,15}$ A successful blockade was indicated by the spread of local anesthetics around the posterior surface of QL muscle under the ultrasound. Another injection was performed identically on the other side. A total of $40 \mathrm{~mL}$ $0.375 \%$ ropivacaine $(150 \mathrm{mg})$ was administered bilaterally in both the ESP and QL blocks. Dosing guidelines (volume and type of local anesthesia) were based on a combination of existing evidence and institutional experience. , $^{4,16}$

\section{Intraoperative Management}

General anesthesia was induced with intravenous (IV) thiopental (4-6 mg $\left.\cdot \mathrm{kg}^{-1}\right)$ and rocuronium $(0.6-0.8 \mathrm{mg} \cdot \mathrm{kg}$ -

${ }^{-1}$ ) followed by tracheal intubation. Anesthesia was maintained with sevoflurane in a 1:1 oxygen:air mixture and IV remifentanil $\left(0.01-0.05 \mu \mathrm{g} \cdot \mathrm{kg}^{-1} \cdot \mathrm{min}^{-1}\right)$ infusion. The mean arterial blood pressure was maintained above 65 mmHg. ${ }^{17}$ All patients received IV meperidine $0.5 \mathrm{mg} \cdot \mathrm{kg}^{-1} 30 \mathrm{~min}$ before the end of surgery in accordance with the institutional protocol. ${ }^{3,4,8}$ The same surgical team performed all surgical procedures (Gyu-Seung Choi or Jong Man Kim).

\section{Postoperative Post-Anesthesia Care Unit and Postoperative Pain Management}

After surgery, patients were transferred to the postanesthesia care unit (PACU). Resting pain severity was assessed by the PACU nurse using an 11-point numeric rating scale (NRS; $0=$ no pain and $10=$ worst pain). All patients received a standardized postoperative supplemental analgesic regimen. ${ }^{3,4,8}$ IV patient-controlled analgesia (PCA) with fentanyl was initiated at the first complaint of surgical site pain (NRS $\geq 1 / 10)$, which deliver a bolus dose of $15 \mu \mathrm{g}(1 \mathrm{~mL})$ of fentanyl and a lockout time of $15-\mathrm{min}$, without background infusion. If the NRS was more than 4/ 10, IV meperidine $25 \mathrm{mg}$ was administered as first-line therapy in the PACU. IV hydromorphone 0.08-0.1 $\mu \mathrm{g} \cdot \mathrm{kg}^{-1}$ was administered as second-line therapy. Postoperative nausea or vomiting was treated with IV metoclopramide $10 \mathrm{mg}$. A PACU nurse recorded all PACU data, including opioid consumption, nausea or vomiting, and pain scores on admission, at discharge, and the highest reported pain score during the PACU stay.

In the general ward, all patients received multimodal analgesia consisting of (1) $400 \mathrm{mg}$ of IV ibuprofen (Huons, Seoul, South Korea) every $6 \mathrm{~h}$ (maximum 6 doses); (2) one tablet of oral C.I.A capsule ${ }^{\circledR}$ (codeine phosphate $10 \quad \mathrm{mg}$ /ibuprofen $200 \mathrm{mg} /$ acetaminophen 250 mg; Myungmoon Pharmacy, Seoul, South Korea) every $8 \mathrm{~h}$; (3) IV fentanyl PCA; and (4) $1 \mathrm{mg}$ of IV hydromorphone every $4 \mathrm{~h}$ as needed as rescue analgesia., ${ }^{3,4,8}$

\section{Outcome and Outcome Measures}

The primary outcome used to assess the quality of postoperative analgesia was cumulative opioid consumption at $24 \mathrm{~h}$ postoperatively. This was calculated as the sum of all rescue opioid doses plus total IV PCA usage and converted into IV morphine equivalents (IME). ${ }^{18}$ Secondary outcomes included (1) resting pain scores in the PACU (admission, highest, and discharge) and, at 24, 48, and 72 h postoperatively; (2) cumulative opioid consumption in the PACU and at 48 and $72 \mathrm{~h}$ postoperatively; (3) 
incidence of postoperative nausea or vomiting in the PACU and at 24, 48, and $72 \mathrm{~h}$ postoperatively; (4) quality of sleep on the first night using a Likert scale $(1=$ very dissatisfied, $2=$ dissatisfied, $3=$ neutral, $4=$ satisfied, and $5=$ very satisfied); (5) patient satisfaction with pain relief at $24 \mathrm{~h}$ postoperatively using a Likert scale; (6) Quality of Recovery (QoR)-15 questionnaire scores preoperatively and $24 \mathrm{~h}$ postoperatively (using the Korean version of the Quality of Recovery-15 scale ${ }^{19}$ ), which can be divided into two components: physical and mental well-being; ${ }^{20}$ (7) the incidence of procedure-related complication (hematoma, infection, or needle trauma); (8) time to first flatus; (9) intraoperative remifentanil consumption; (10) block performance time defined as time from insertion of the needle to completion of the block; and (11) total arterial plasma ropivacaine concentrations at $30,45,60$, and 240 min after local anesthetic injection. An independent outcome assessor visited each patient at predetermined time points $(24,48$, and $72 \mathrm{~h}$ postoperatively) to collect all outcome data.

\section{Total Arterial Plasma Ropivacaine Concentration Analysis}

The right radial artery was accessed using a 20-gauge catheter after induction of general anesthesia. Arterial blood samples were collected from the indwelling arterial catheter at 30, 45, 60, and $240 \mathrm{~min}$ after the second dose of ropivacaine administration. ${ }^{9}$ According to the initial investigational plan, ropivacaine concentrations were measured at baseline (pre-block), and 30, 60, and $240 \mathrm{~min}$, however, the sampling protocol was modified to include additional samples at $45 \mathrm{~min}$ instead of baseline to better define the peak concentration of ropivacaine. The modified protocol was approved by the Samsung Medical Center Research Ethics Board (SMC 2020-01-035-010) and reported to the CRiS trial registry of South Korea. The samples were stored at $4{ }^{\circ} \mathrm{C}$ and delivered to the clinical laboratory within $1 \mathrm{~h}$ of collection, where they were centrifuged at 2500 revolutions $/ \mathrm{min}$ for $5 \mathrm{~min}$ at $4{ }^{\circ} \mathrm{C}$ and transferred to a $-70{ }^{\circ} \mathrm{C}$ freezer for storage prior to transport to the SCL healthcare laboratory (Seoul, South Korea) for analysis. Total plasma ropivacaine concentration (measuring the sum of bound and unbound fractions) was analyzed using liquid chromatography-tandem mass spectrometry (API 4000 LC-MS/MS system, Applied Biosystems, USA) with a chromatography column. The detection limit was $5 \mathrm{ng} / \mathrm{mL}$. The internal standard used was
Ropivacaine-D7 (Santa Cruz Biotechnology, USA). A previous report presented an arterial threshold value of total ropivacaine of $4.3 \pm 0.6 \mu \mathrm{g} / \mathrm{mL}$ for systemic toxicity. $^{21}$

\section{Statistical Analysis}

Sample Size Calculations

This study was designed as a superiority trial. Based on a previous study ${ }^{3}$ and retrospective data from our experience, we calculated the mean \pm standard deviation for cumulative opioid consumption at $24 \mathrm{~h}$ to be $45.5 \pm$ $21.8 \mathrm{mg}$ IME. A $30 \%$ reduction in the mean cumulative 24-h opioid consumption after bilateral QL blocks compared to that with the bilateral ESP blocks was considered clinically significant. Sample size calculation using G*Power (v. 3.1.9.4), with $\alpha=0.05$ and power of $80 \%$ showed that 42 patients were required in each group. We decided to enroll 44 patients per group to allow for dropouts $(5 \%)$.

\section{Data Analyses}

After determining the normality of data distributions using the Shapiro-Wilk test, continuous variables were analyzed using the $t$-test or Mann-Whitney $U$-test as appropriate. Parametric and non-parametric data are reported as mean \pm standard deviation and median [interquartile ranges], respectively. Categorical variables were analyzed using the chi-square test or Fisher's exact test. For all outcomes with repeated measures (cumulative opioid consumption, pain scores, incidence of postoperative nausea or vomiting, and ropivacaine concentrations), Bonferroni correction was applied for multiple comparisons. The peak plasma concentration of ropivacaine $\left(\mathrm{C}_{\max }\right)$ and time to $\mathrm{C}_{\max }\left(\mathrm{T}_{\max }\right)$ were obtained directly from the observed concentration-time data, which are summarized as mean \pm standard deviation and median [interquartile ranges], respectively. Data analyses were performed using SPSS software (ver. 27.0; SPSS Inc., Chicago, IL). Two-sided tests were used for all analyses, and $P<0.05$ was taken to indicate significance. All analyses were conducted using an intention-to-treat approach.

\section{Results}

\section{Study Participants}

Eighty-eight patients scheduled for laparoscopic liver resection between December 2020 and July 2021 were assessed 


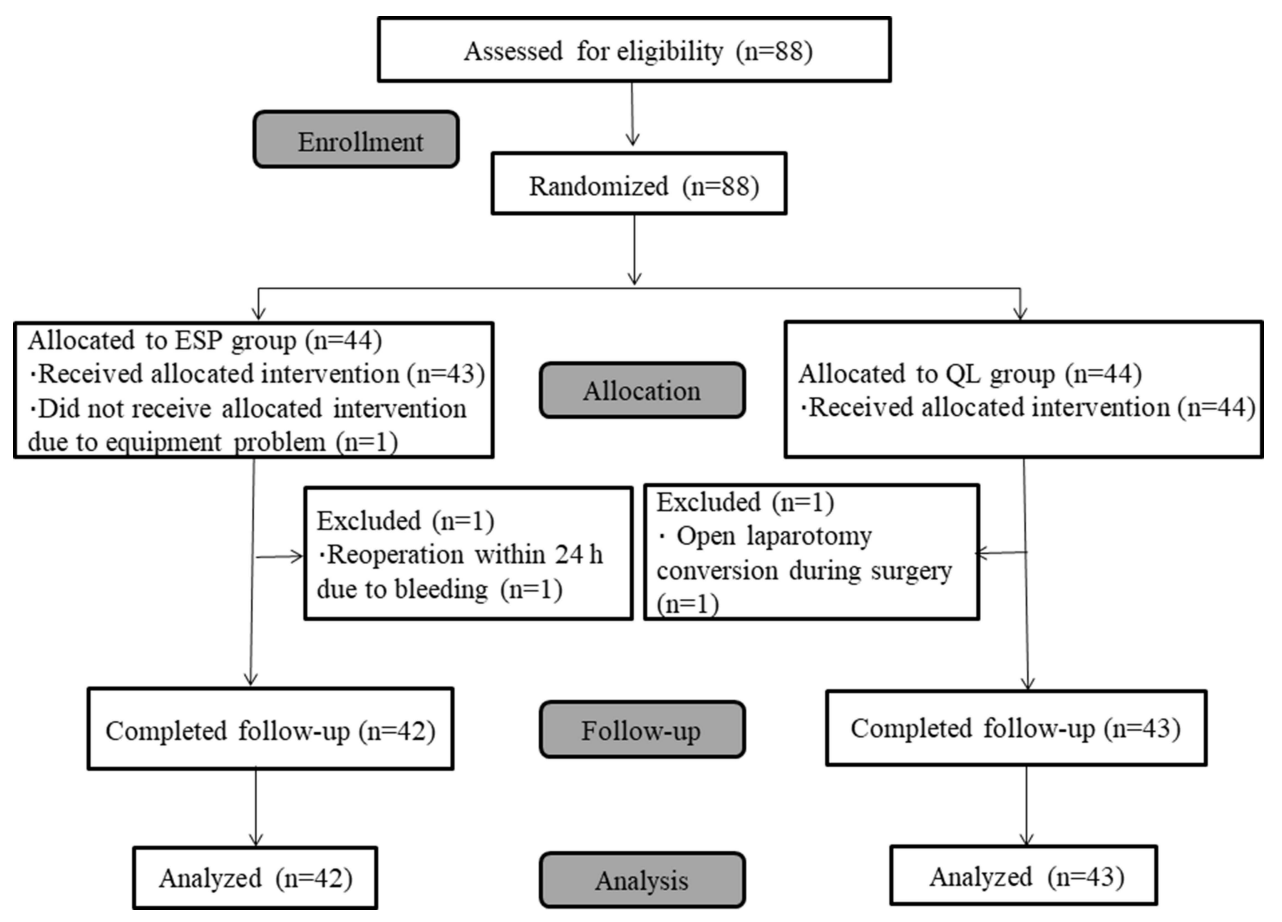

Figure I Consolidated standards of reporting trials flow diagram showing patient progress through the study phases. Abbreviations: ESP, erector spinae plane; QL, quadratus lumborum.

for eligibility (Figure 1). All enrolled patients $(\mathrm{n}=88)$ were randomly assigned to one of two groups ( $\mathrm{n}=44$ each). One patient in the ESP group did not receive the allocated intervention due to equipment problems, and one patient in the ESP group received allocated intervention but underwent reoperation within $24 \mathrm{~h}$ after surgery due to postoperative bleeding. One patient in the QL group received the allocated intervention but underwent open laparotomy conversion during surgery. These three patients were excluded from further analyses. A total of 85 patients were analyzed (ESP group, $n=42$; $Q L$ group, $n=43$ ). Baseline patient and surgical characteristics of the two groups were comparable, except for the significantly higher score in the QoR-15 questionnaire in the ESP group than in the QL group because the ESP group had significantly higher physical well-being scores than the QL group (Table 1). This difference was because the ESP group had significantly higher physical well-being scores than those of the QL group.

Table I Patients' Characteristics in the Erector Spinae Plane (ESP) Block Group and Quadratus Lumborum (QL) Block Group

\begin{tabular}{|c|c|c|c|}
\hline Parameter & $\operatorname{ESP}(n=42)$ & QL $(n=43)$ & $P$ value \\
\hline Age (years) & $52.7 \pm 11.9$ & $52.6 \pm 9.4$ & 0.954 \\
\hline Sex (male/female) & $27 / 15$ & $30 / 13$ & 0.649 \\
\hline Body mass index $\left(\mathrm{kg} \mathrm{m}^{2}\right)$ & $24.6 \pm 3.4$ & $24.8 \pm 4.1$ & 0.769 \\
\hline ASA physical status (I/II/III) & $4 / 33 / 5$ & $9 / 33 / 1$ & 0.101 \\
\hline QoR-I5 scores at preoperatively (score range from 0 to 150 ) & $142.8 \pm 7.7$ & $138.4 \pm 10.5$ & 0.029 \\
\hline Physical well-being (score range from 0 to 90 ) & $87.9 \pm 2.9$ & $85.5 \pm 5.7$ & 0.019 \\
\hline Mental well-being (score range from 0 to 60 ) & $55.0 \pm 5.8$ & $52.9 \pm 5.7$ & 0.101 \\
\hline Block procedure time (minutes) & $2.0 \pm 0.6$ & $2.2 \pm 0.9$ & 0.697 \\
\hline Duration of anesthesia (hours) & $3.1 \pm 0.8$ & $3.3 \pm 1.0$ & 0.546 \\
\hline Duration of surgery (hours) & $2.4 \pm 0.7$ & $2.5 \pm 1.0$ & 0.558 \\
\hline Duration of PACU stay (minutes) & $58.6 \pm 9.6$ & $58.2 \pm 9.3$ & 0.875 \\
\hline
\end{tabular}

Note: Values are mean \pm standard deviations or number.

Abbreviations: PACU, post-anesthesia care unit; QoR, Quality of recovery. 
Table 2 Differences in Cumulative Opioid Consumption in IV Morphine Equivalent (mg) Between the Erector Spinae Plane (ESP) Block Group and Quadratus Lumborum (QL) Block Group

\begin{tabular}{|l|c|c|c|c|}
\hline Time Points & ESP $(\mathbf{n}=\mathbf{4 2})$ & QL $(\mathbf{n}=\mathbf{4 3})$ & Mean Differencesł (QL-ESP) (95\% Cl) & P value \\
\hline At PACU & $4.3 \pm 2.1$ & $3.8 \pm 2.2$ & $-0.5(-1.4$ to 0.4$)$ & $>0.99 *$ \\
At $24 \mathrm{~h}$ & $41.4 \pm 22.6$ & $44.2 \pm 20.0$ & $2.8(-6.4$ to 12.0$)$ & $>0.99 *$ \\
At $48 \mathrm{~h}$ & $70.9 \pm 40.6$ & $75.2 \pm 35.4$ & $4.3(-12.1$ to 20.8$)$ & $>0.99 *$ \\
At $72 \mathrm{~h}$ & $140.9 \pm 81.7$ & $158.6 \pm 74.1$ & $17.7(-16$ to 51.3$)$ & $>0.99 *$ \\
\hline
\end{tabular}

Notes: Values are mean \pm standard deviations and mean differences with $95 \%$ confidence interval $(\mathrm{Cl})$. The $P$ value for the $t$-test is set at 0.05 . Individual $* P$ values result from a Bonferroni correction for multiple comparisons.

\section{Cumulative Opioid Consumption}

The primary outcome of cumulative opioid consumption at $24 \mathrm{~h}$ postoperatively was not significantly different between the two groups (ESP vs QL group; $41.4 \pm 22.6 \mathrm{mg}$ vs $44.2 \pm$ $20.0 \mathrm{mg}$ IME, mean difference [QL-ESP), $2.8 \mathrm{mg}, 95 \% \mathrm{CI}$, -6.4 to $12 \mathrm{mg}$ IME, $\mathrm{p}>0.99$ ) (Table 2). There was no significant difference in cumulative opioid consumption at 48 and 72 h postoperatively ( $>0.99$ ).

\section{Pain Score and Other Secondary Outcomes}

Intraoperative remifentanil consumption and the incidence of intraoperative hypotension were similar between the groups (Table 3). There was no significant difference in resting pain scores in the PACU (admission, highest, and discharge) and, at 24,48 , and $72 \mathrm{~h}$ postoperatively (Figure 2). There was no significant difference between groups in terms of quality of sleep on the first postoperative night, patient satisfaction with pain relief at $24 \mathrm{~h}$ postoperatively, QoR-15 scores at $24 \mathrm{~h}$ postoperatively, including physical and mental wellbeing, time to first flatus, or the incidence of postoperative nausea or vomiting (Table 3). No block-related complications were reported in either group.

\section{Total Arterial Plasma Ropivacaine Concentrations}

Blood tests of the first (30 $\mathrm{min})$ and third $(60 \mathrm{~min})$ panels of the ropivacaine assay were completed according to the

Table 3 Perioperative Secondary Outcomes in the Erector Spinae Plane (ESP) Block Group and the Quadratus Lumborum (QL) Block Group

\begin{tabular}{|c|c|c|c|}
\hline Outcomes & $\operatorname{ESP}(n=42)$ & QL $(n=43)$ & $\mathbf{P}^{*}$ \\
\hline Intraoperative remifentanil consumption $\left(\mu \mathrm{g} \mathrm{kg}^{-1} \mathrm{~min}^{-1}\right)$ & $0.03[0.00-0.05]$ & $0.03[0.01-0.04]$ & 0.109 \\
\hline Intraoperative hypotension, $\mathrm{n}(\%)$ & $15(35.7 \%)$ & $13(30.2 \%)$ & 0.591 \\
\hline Quality of sleep on the first night (Likert scale; $I$ to $5^{\dagger}$ ) & $4[2-4.3]$ & $4[3-4]$ & 0.319 \\
\hline \multicolumn{4}{|l|}{ Patient satisfaction with pain relief at $24 \mathrm{~h}, \mathrm{n}$} \\
\hline Very dissatisfied/dissatisfied/ neutral/satisfied/very satisfied & $0 / 5 / 7 / 21 / 9$ & $2 / 0 / 7 / 26 / 8$ & 0.108 \\
\hline QoR-15 scores at $24 \mathrm{~h}$ (score range from 0 to 150 ) & $103.7 \pm 27.1$ & $100.5 \pm 32.2$ & 0.621 \\
\hline Physical well-being (score range from 0 to 90 ) & $55.6 \pm 17.5$ & $55.1 \pm 20.3$ & 0.921 \\
\hline Mental well-being (score range from 0 to 60 ) & $48.1 \pm 11.5$ & $45.3 \pm 13.2$ & 0.302 \\
\hline Time to first flatus (hours) & $51.4 \pm 19.5$ & $50.8 \pm 16.2$ & 0.856 \\
\hline \multicolumn{4}{|l|}{ Postoperative nausea or vomiting, $n$ (\%) } \\
\hline At $24 \mathrm{~h}$ & 7 (16.7\%) & $14(32.6 \%)$ & $0.267^{*}$ \\
\hline At $48 \mathrm{~h}$ & $12(28.6 \%)$ & $14(32.6 \%)$ & $>0.99 *$ \\
\hline At $72 \mathrm{~h}$ & 5 (II.9\%) & 9 (20.9\%) & $0.786^{*}$ \\
\hline \multicolumn{4}{|l|}{ Patients requiring metoclopramide, $\mathrm{n}(\%)$} \\
\hline $0-24$ hours & $12(28.6 \%)$ & $10(23.3 \%)$ & $>0.99 *$ \\
\hline 24-48 hours & $9(21.4 \%)$ & II (25.6\%) & $>0.99 *$ \\
\hline $48-72$ hours & $5(11.9 \%)$ & $6(14 \%)$ & $>0.99 *$ \\
\hline Block related complications, n (\%) & 0 & 0 & N/A \\
\hline
\end{tabular}

Notes: Values are mean \pm standard deviations, median [interquartile ranges], or number (percentage). The $P$ value for the $t$-test, Mann-Whitney test, or Fisher's exact test is set at 0.05 . Individual $* P$ values result from a Bonferroni correction for multiple comparisons. 'Likert scale where I=very dissatisfied, $2=$ dissatisfied, $3=$ neutral, $4=$ satisfied and $5=$ very satisfied.

Abbreviations: N/A, not applicable; QoR, Quality of Recovery. 


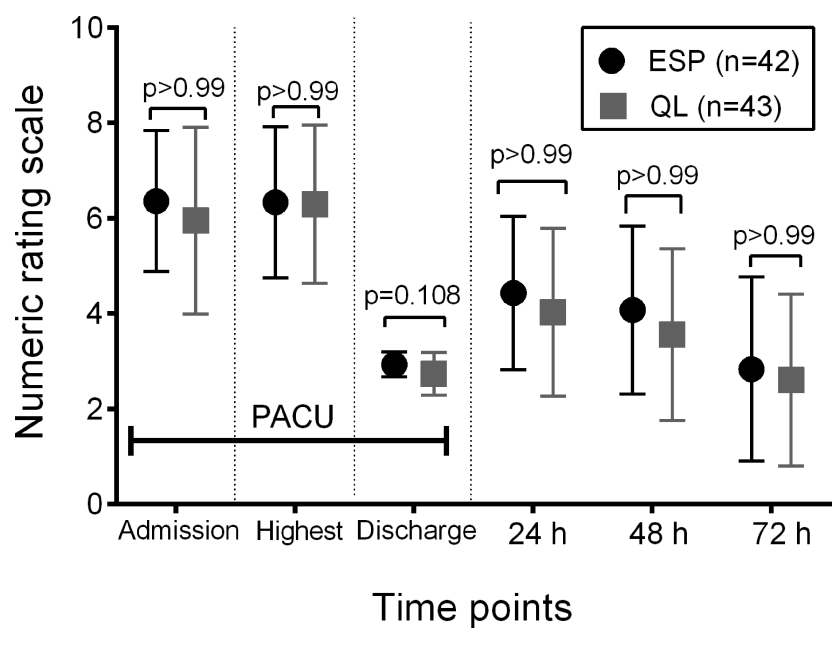

Figure 2 Numeric rating scale (NRS) scores at rest for the two groups within 72 hours postoperatively. $P$ values result from a Bonferroni correction for multiple comparisons.

Abbreviations: ESP, erector spinae plane; QL, quadratus lumborum.

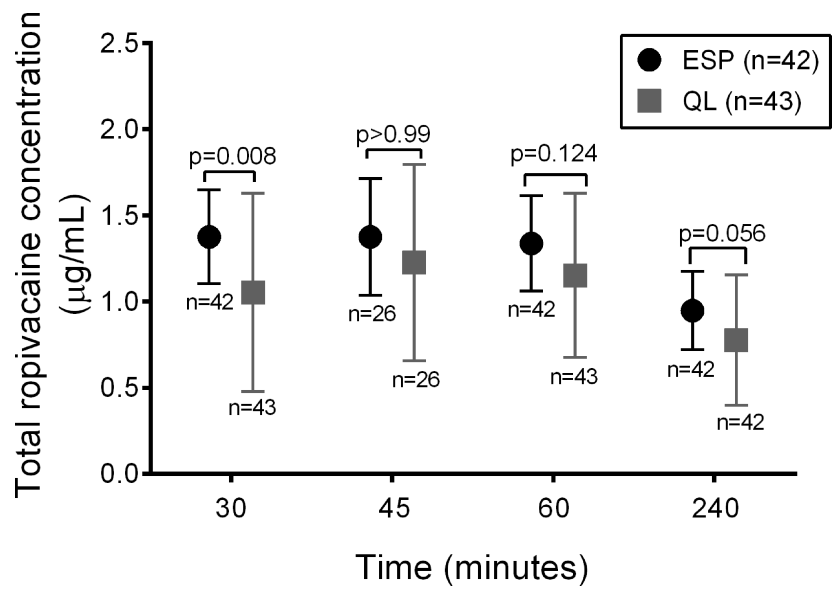

Figure 3 Chronological changes in total arterial plasma ropivacaine concentrations after blockade. $P$ values result from a Bonferroni correction for multiple comparisons.

Abbreviations: ESP, erector spinae plane; QL, quadratus lumborum.

designated timing in the study protocol. The second (45 min) panel of ropivacaine assays was obtained in 26 patients in the ESP group and 26 patients in the QL group due to changes in the blood sampling protocol during the study. The last panel (240 min) of ropivacaine assays was not obtained in one patient in the QL group because the timing of the tests was not in accordance with the study protocol. Total ropivacaine concentration at 30 min was significantly higher in the ESP group than in the QL group (ESP vs QL block; $1.4 \pm 0.3$ vs $1.1 \pm 0.6 \mu \mathrm{g}$ / $\mathrm{mL}, \mathrm{p}=0.008$ ) (Figure 3). The $\mathrm{C}_{\max }$ was significantly higher in the ESP group $(1.5 \pm 0.3 \mu \mathrm{g} / \mathrm{mL})$ than in the
QL group $(1.3 \pm 0.5 \mu \mathrm{g} / \mathrm{mL}, \mathrm{p}=0.035)$, but both were lower than the arterial threshold value of systemic toxicity $(4.3 \mu \mathrm{g} / \mathrm{mL})$. The $\mathrm{T}_{\max }$ was comparable between the two groups (ESP vs QL group: 45 [30-48.8] vs 45 [30-60] min, $\mathrm{p}=0.295$ ). All the test results were below the documented toxic concentration of $4.3 \mu \mathrm{g} / \mathrm{mL}$. No adverse events or clinical symptoms indicating systemic toxicity were observed.

\section{Discussion}

In this randomized controlled trial comparing bilateral single-injection ESP blocks with bilateral single-injection posterior QL blocks, we were unable to demonstrate a difference between groups in the primary outcome of cumulative opioid consumption during the first $24 \mathrm{~h}$ after laparoscopic liver resection. There were no significant differences in pain scores or opioid consumption between the two blocks at 48 and $72 \mathrm{~h}$ postoperatively. Recovery outcomes, including postoperative nausea or vomiting, QoR-15 scores, sleep quality on the first night, and patient satisfaction, were also similar between the two groups. Plasma ropivacaine concentration at $30 \mathrm{~min}$ after $150 \mathrm{mg}$ of ropivacaine injection was significantly lower in the QL group than in the ESP group, however, all measurements were below the cut-off value $(<4.3 \mu \mathrm{g} / \mathrm{mL})$ for the risk of systemic toxicity of arterial total ropivacaine concentrations.

The ESP block has emerged as a potentially useful regional anesthesia technique in abdominal surgery. ${ }^{5,6}$ Our previous study also demonstrated the adequate analgesic efficacy of bilateral single-injection ESP blocks in donors who underwent laparoscopic donor hepatectomy. ${ }^{4}$ However, a recent study conducted at our institution in patients with HCC demonstrated that ESP block decreased rescue opioid dose in PACU but did not reduce opioid consumption in the first $24 \mathrm{~h}$ after laparoscopic liver resection compared to that with the systemic control. ${ }^{3}$ The analgesic effect of the ESP block differed between the two studies, despite being conducted in the same center and the same surgical team, might be ascribed to the differences in the detailed perioperative analgesic methodology (ESP block injection point [T8 vs T9] and IV PCA regimen) and patient characteristics (young healthy donors vs middle-aged HCC patients). The severity of postoperative pain and opioid requirements in patients with chronic liver disease was different from that in other patients. ${ }^{22,23}$ In the current study, the pain scores and cumulative opioid consumption in the ESP group were similar to those in 
a previous study. ${ }^{3}$ Therefore, it can be deduced that both ESP and QL blocks may provide mainly intraoperative and immediate postoperative analgesia, as evidenced by an opioid-sparing effect in the PACU.

The QL block has been increasingly used for postoperative analgesia in various types of surgery including cesarean section, ${ }^{13}$ laparoscopic ovarian surgery, ${ }^{9}$ hip arthroplasty, ${ }^{24}$ and laparoscopic hepatectomy. ${ }^{12}$ Its analgesic effect is ascribed to the spread of local anesthetic injected between the transversalis fascia and the quadratus lumborum muscle to the thoracic paravertebral space, thus, anesthetizing the thoracolumbar nerves. ${ }^{10}$ Previous studies demonstrated that the effect of a single-injection of posterior QL block with $20 \mathrm{~mL}$ of $0.375 \%$ ropivacaine could spread from T4 to T12 or L1 ${ }^{9,10}$ and could last up to 48 $\mathrm{h}$ postoperatively, ${ }^{9,14}$ which is relatively longer than that with single-injection ESP block (5-12 h) ${ }^{7,25}$ Based on this, we hypothesized that the posterior QL block would provide a prolonged analgesic effect, and thus, reduce the cumulative opioid consumption within $24 \mathrm{~h}$ compared to the ESP block. However, the results of our study did not demonstrate the superiority of the analgesic effect of the QL block over that of the ESP block, which is consistent with a recent procedure specific postoperative pain management (PROSPECT) review that found no evidence to support QL block after open liver resection. ${ }^{26}$

The ease of performance and risk of local anesthetic systemic toxicity are important in daily practice. In our clinical experience with ESP block, changing the patient's position from supine to lateral for the block procedure requires multiple personnel and increases the risk of falls, especially in anesthetized patients. In contrast, the QL block can be performed with the patient in a supine position. However, in obese patients, it is difficult to obtain high-quality ultrasound images and placing a thin pillow under the patient's hip or lateral tilt improves the images in these cases. In our study, the patient was supine, but depending on the physician's preference and patient mobility, the patient can lie lateral, sitting or prone. ${ }^{10}$ Therefore, QL block can be a favorable option in terms of ease of performance and practicality over ESP block. The major risk associated with the fascial plane block is local anesthetic systemic toxicity due to systemic absorption. ${ }^{27}$ The instances of local anesthetic systemic toxicity following the ESP block have been minor to date. $^{28,29}$ In addition, no systemic toxicity was observed after the QL block with $150 \mathrm{mg}$ of ropivacaine. ${ }^{9}$ Notably, ropivacaine is mainly eliminated by hepatic metabolism, with reduced clearance in patients with chronic liver disease. ${ }^{30}$ However, in our study, the peak ropivacaine concentrations after the ESP or QL blocks were below the arterial threshold of systemic toxicity $(4.3 \mu \mathrm{g} / \mathrm{mL}) .{ }^{21}$ Moreover, ropivacaine concentrations at $240 \mathrm{~min}$ after injection were also below the systemic toxicity threshold in both groups, and thus, it can be deduced that the metabolism of $150 \mathrm{mg}$ of ropivacaine is not hindered even after removal of a significant portion of liver mass. Although free plasma ropivacaine concentrations were not measured in this study, our results suggest that both ESP and QL blocks with $150 \mathrm{mg}$ of ropivacaine might be performed safely in patients undergoing liver surgery. This is also supported by the fact that there were no patients with any clinical signs or symptoms of local systemic anesthetic toxicity. The peak ropivacaine concentration was significantly lower in the QL group than in the ESP group, and the difference in peak ropivacaine concentration between the two blocks could be explained by the fact that the rate of local anesthetic absorption into the blood depends on the local tissue perfusion. ${ }^{31}$ Epinephrine added to ropivacaine decreased peak plasma concentrations and delayed the time of peak concentration in thoracic paravertebral block $^{32}$ or posterior QL block. ${ }^{33}$ Although epinephrine was not added to ropivacaine in this study, the risk of systemic toxicity would likely be lower if epinephrine was added, and further study is needed to confirm this. Since the local anesthetic was injected within recommended doses $(0.2-0.4 \mathrm{~mL} / \mathrm{kg}$ of $0.2-0.5 \%$ ropivacaine $),{ }^{10}$ our results for ropivacaine concentration can be generalized to a variety of clinical settings.

Several study limitations should be considered when interpreting our results. First, we did not have a control group that received systemic analgesia alone or placebo, which would have further defined the benefit afforded by ESP or QL blocks. However, the institutional ethics board prevented us from using placebo or sham blocks. Second, we only assessed resting pain scores. An assessment of pain during movement might have uncovered further functional differences in analgesic quality between the groups. Third, we did not assess block success by evaluation of dermatomal sensory loss using pinprick test or cold alcohol swab, which may have contributed to the observed results. Fourth, we only measured total plasma ropivacaine concentrations. The toxicity of ropivacaine arises from the unbound fraction of ropivacaine. ${ }^{21}$ Therefore, we cannot rule out the possibility that our data underestimated or 
overestimated the plasma concentration for toxicity. Finally, it is conceivable that the use of different types of QL block (eg, lateral or anterior), different volumes and doses of local anesthesia, or other combinations of multimodal analgesics will produce different outcomes, as the optimal regimen for ESP block or QL block in laparoscopic liver resection has yet to be determined.

\section{Conclusion}

In conclusion, bilateral single-injection QL block did not reduce cumulative opioid consumption over 24 $\mathrm{h}$ compared to the bilateral single-injection ESP block in patients undergoing laparoscopic liver resection. Further studies are needed to define the benefits afforded by ESP or QL blocks in patients undergoing liver surgery.

\section{Disclosure}

Prof. Dr. RyungA Kang report grants from Samsung Medical Center Grant \# SMO1210471. The authors report no other conflicts of interest in this work.

\section{References}

1. Kim JM, Kwon CHD, Yoo H, et al. Which approach is preferred in left hepatocellular carcinoma? Laparoscopic versus open hepatectomy using propensity score matching. BMC Cancer. 2018;18(1):668 doi:10.1186/s12885-018-4506-3

2. Jeong JS, Wi W, Chung YJ, et al. Comparison of perioperative outcomes between pure laparoscopic surgery and open right hepatectomy in living donor hepatectomy: propensity score matching analysis. Sci Rep. 2020;10(1):5314. doi:10.1038/s41598-020-62289-0

3. Kim D, Kim JM, Choi GS, Heo G, Kim GS, Jeong JS. Ultrasoundguided erector spinae plane block for postoperative analgesia in laparoscopic liver resection: a prospective, randomised controlled, patient and observer-blinded study. Eur J Anaesthesiol. 2021;38 (Suppl 2):S106-S112. doi:10.1097/EJA.0000000000001475

4. Kang R, Chin KJ, Gwak MS, et al. Bilateral single-injection erector spinae plane block versus intrathecal morphine for postoperative analgesia in living donor laparoscopic hepatectomy: a randomized non-inferiority trial. Reg Anesth Pain Med. 2019:rapm-2019-100902. doi:10.1136/rapm-2019-100902

5. Chin KJ, Malhas L, Perlas A. The erector spinae plane block provides visceral abdominal analgesia in bariatric surgery. Reg Anesth Pain Med. 2017;42(3):372-376. doi:10.1097/aap.0000000000000581

6. Chin KJ, Adhikary S, Sarwani N, Forero M. The analgesic efficacy of pre-operative bilateral erector spinae plane (ESP) blocks in patients having ventral hernia repair. Anaesthesia. 2017;72(4):452-460. doi:10.1111/anae.13814

7. Abu Elyazed MM, Mostafa SF, Abdelghany MS, Eid GM. Ultrasoundguided erector spinae plane block in patients undergoing open epigastric hernia repair: a prospective randomized controlled study. Anesth Analg. 2019;129(1):235-240. doi:10.1213/ANE.000 0000000004071

8. Kang R, Chin KJ, Kim GS, et al. Bilateral continuous erector spinae plane block using a programmed intermittent bolus regimen versus intrathecal morphine for postoperative analgesia in living donor laparoscopic hepatectomy: a randomized controlled trial. J Clin Anesth. 2021;75:110479. doi:10.1016/j.jclinane.2021.110479
9. Murouchi T, Iwasaki S, Yamakage M. Quadratus lumborum block: analgesic effects and chronological ropivacaine concentrations after laparoscopic surgery. Reg Anesth Pain Med. 2016;41(2):146-150. doi:10.1097/AAP.0000000000000349

10. Elsharkawy H, El-Boghdadly K, Barrington M. Quadratus lumborum block: anatomical concepts, mechanisms, and techniques. Anesthesiology. 2019;130(2):322-335. doi:10.1097/ALN.00000000 00002524

11. Zhu Q, Li L, Yang Z, et al. Ultrasound guided continuous quadratus lumborum block hastened recovery in patients undergoing open liver resection: a randomized controlled, open-label trial. $B M C$ Anesthesiol. 2019;19(1):23. doi:10.1186/s12871-019-0692-Z

12. Pang M, Sun G, Yao W, et al. Ultrasound-guided transmuscular quadratus lumborum block reduced postoperative opioids consumptions in patients after laparoscopic hepatectomy: a three-arm randomized controlled trial. BMC Anesthesiol. 2021;21(1):45. doi:10.1186/ s12871-021-01255-3

13. Blanco R, Ansari T, Girgis E. Quadratus lumborum block for postoperative pain after caesarean section: a randomised controlled trial. Eur J Anaesthesiol. 2015;32(11):812-818. doi:10.1097/EJA.00000 00000000299

14. Blanco R, Ansari T, Riad W, Shetty N. Quadratus lumborum block versus transversus abdominis plane block for postoperative pain after cesarean delivery: a randomized controlled trial. Reg Anesth Pain Med. 2016;41(6):757-762. doi:10.1097/AAP.0000000000000495

15. Kariem El-Boghdadly HE, Short A, Chin KJ. Quadratus lumborum block nomenclature and anatomical considerations. Reg Anesth Pain Med. 2016;41:549.

16. Lee SH, Gwak MS, Choi SJ, et al. Prospective, randomized study of ropivacaine wound infusion versus intrathecal morphine with intravenous fentanyl for analgesia in living donors for liver transplantation. Liver Transpl. 2013;19(9):1036-1045. doi:10.1002/ 1t.23691

17. Lee EK, Kim J, Ko JS, Gwak MS, Kim G. Symptomatic (hypotensive) bradycardia during laparoscopic living donor hepatectomy: incidence and risk factors. Transplant Proc. 2020;52(6):1788-1790. doi:10.1016/j.transproceed.2020.01.161

18. Scott M, Fishman JCB, Rathmell James P. Bonica's Management of Pain. 4th ed. Philadelphia: Lippincott Williams \& Wilkins; 2010.

19. Yoon S, Joo H, Oh YM, Lee J, Bahk JH, Lee HJ. Validation and clinical utility of the Korean version of the quality of recovery-15 with enhanced recovery after surgery: a prospective observational cohort study. Br J Anaesth. 2020;125(4):614-621. doi:10.1016/j. bja.2020.06.040

20. Stark PA, Myles PS, Burke JA. Development and psychometric evaluation of a postoperative quality of recovery score: the QoR-15. Anesthesiology. 2013;118(6):1332-1340. doi:10.1097/ALN.0b013e $318289 \mathrm{~b} 84 \mathrm{~b}$

21. Knudsen K, Beckman Suurkula M, Blomberg S, Sjovall J, Edvardsson N. Central nervous and cardiovascular effects of i.v. infusions of ropivacaine, bupivacaine and placebo in volunteers. $\mathrm{Br}$ J Anaesth. 1997;78(5):507-514. doi:10.1093/bja/78.5.507

22. Ko JS, Shin YH, Gwak MS, Jang CH, Kim GS, Lee SK. The relationship between postoperative intravenous patient-controlled fentanyl analgesic requirements and severity of liver disease. Transplant Proc. 2012;44(2):445-447. doi:10.1016/j.transpr oceed.2012.01.017

23. Chen JP, Jawan B, Chen CL, et al. Comparison of postoperative morphine requirements in healthy living liver donors, patients with hepatocellular carcinoma undergoing partial hepatectomy, and liver transplant recipients. Transplant Proc. 2010;42(3):701-702. doi:10.1016/j.transproceed.2010.02.024

24. Brixel SM, Biboulet P, Swisser F, et al. Posterior quadratus lumborum block in total hip arthroplasty: a randomized controlled trial. Anesthesiology. 2021;134(5):722-733. doi:10.1097/ALN.000000000 0003745 
25. Gurkan Y, Aksu C, Kus A, Yorukoglu UH, Kilic CT. Ultrasound guided erector spinae plane block reduces postoperative opioid consumption following breast surgery: a randomized controlled study. J Clin Anesth. 2018;50:65-68. doi:10.1016/j.jclinane.2018.06.033

26. Dieu A, Huynen P, Lavand'homme P, et al. Pain management after open liver resection: Procedure-Specific Postoperative Pain Management (PROSPECT) recommendations. Reg Anesth Pain Med. 2021;46(5):433-445. doi:10.1136/rapm-2020-101933

27. Elsharkawy H, Pawa A, Mariano ER. Interfascial plane blocks: back to basics. Reg Anesth Pain Med. 2018;43(4):341-346. doi:10.1097/ AAP.0000000000000750

28. Tulgar S, Selvi O, Senturk O, Serifsoy TE, Thomas DT. Ultrasoundguided erector spinae plane block: indications, complications, and effects on acute and chronic pain based on a single-center experience. Cureus. 2019;11(1):e3815. doi:10.7759/cureus.3815

29. De Cassai A, Geraldini F, Carere A, Sergi M, Munari M. Complications rate estimation after thoracic erector spinae plane block. J Cardiothorac Vasc Anesth. 2021;35(10):3142-3143. doi:10.1053/j.jvca.2021.02.043
30. Jokinen MJ, Neuvonen PJ, Lindgren L, et al. Pharmacokinetics of ropivacaine in patients with chronic end-stage liver disease. Anesthesiology. 2007;106(1):43-55. doi:10.1097/00000542-2007 01000-00011

31. Thomas JM, Schug SA. Recent advances in the pharmacokinetics of local anaesthetics. Long-acting amide enantiomers and continuous infusions. Clin Pharmacokinet. 1999;36(1):67-83. doi:10.2165/ 00003088-199936010-00005

32. Karmakar MK, Ho AM, Law BK, Wong AS, Shafer SL, Gin T. Arterial and venous pharmacokinetics of ropivacaine with and without epinephrine after thoracic paravertebral block. Anesthesiology. 2005;103(4):704-711. doi:10.1097/00000542-200510000-00008

33. Zanfini BA, Biancone M, Famele M, et al. Comparison of ropivacaine plasma concentration after posterior quadratus lumborum block in cesarean section with ropivacaine with epinephrine vs plane. Minerva Anestesiol. 2021. doi:10.23736/S0375-9393.21.15354-4

\section{Publish your work in this journal}

The Journal of Pain Research is an international, peer reviewed, open access, online journal that welcomes laboratory and clinical findings in the fields of pain research and the prevention and management of pain. Original research, reviews, symposium reports, hypothesis formation and commentaries are all considered for publication. The manuscript management system is completely online and includes a very quick and fair peer-review system, which is all easy to use. Visit http:// www.dovepress.com/testimonials.php to read real quotes from published authors. 